\title{
STATE SPACES FOR MARKOV CHAINS
}

\author{
BY
}

J. L. DOOB

\begin{abstract}
If $p(t, i, j)$ is the transition probability (from $i$ to $j$ in time $t$ ) of a continuous parameter Markov chain, with $p(0+, i, i)=1$, entrance and exit spaces for $p$ are defined. If $L\left[L^{*}\right]$ is an entrance [exit] space, the function $p(\cdot, \cdot, j)[p(\cdot, i, \cdot) / h(\cdot)]$ has a continuous extension to $(0, \infty) \times L\left[(0, \infty) \times L^{*}\right.$, for a certain norming function $h$ on $\left.L^{*}\right]$. It is shown that there is always a space which is both an entrance and exit space. On this space one can define right continuous strong Markov processes, for the parameter interval $[0, b]$, with the given transition function as conditioned by specification of the sample function limits at 0 and $b$.
\end{abstract}

Summary. Let $p$ be a stochastic transition function on the set $I$ of integers: $p(t, i, j)$ is the probability of a transition from $i$ to $j$ in time $t$. An entrance space for $p$ is a topological space $L \supset I$ with the property that $p(\cdot, \cdot, j)$ has a continuous extension to $(0, \infty) \times L$, and that the transition function together with any absolute probability function $f$ determines a Markov process with state space $L$, parameter interval $(0, \infty)$, right continuous with left limits, with limit at 0 if $f=p(\cdot, \xi, \cdot)$ for a point $\xi$ in $L$. An exit space $L^{*}$ is a space which, together with an adjoined isolated point, is an entrance space for a certain (not uniquely determined) dual transition function, and has among other properties the property that, with a certain normalizing function $h$ on $I$, the function $(t, j) \leadsto p(t, i, j) / h(j)$ has a continuous finite extension to $(0, \infty) \times L^{*}$. Previous treatments (see for example [2], [4], [5]) have concentrated attention on a certain entrance space, but in the course of an analysis of entrance and exit spaces it is shown in the present paper that there is a space which is both an entrance and an exit space for $p$. Space time $\alpha$ excessive functions and measures on entrance, exit, and combined spaces are studied. On the appropriate state spaces the natural supermartingales defined in terms of $p$ and its extensions have smooth sample functions. If $L$ is both an entrance and an exit space it is possible to define Markov processes $\{x(t), 0<t<b\}$ with state space $L$, which are Markov processes having the given transition function, conditioned by specification of $x(0+)$ and $x(b-)$.

\section{Introduction.}

(1.1) Standard transition function. Let $p$ be a standard transition function on

Received by the editors December 7, 1969.

AMS Subject Classifications. Primary 6065; Secondary 6062.

Key Words and Phrases. State space compactification, right continuous Markov processes, entrance and exit laws.

Copyright (C) 1970, American Mathematical Society 
the set $I$ of integers, that is a function from $(0, \infty) \times I \times I$ into $[0,1]$ satisfying

$$
\begin{gathered}
\sum_{j} p(t, i, j)=1, \quad \lim _{t \rightarrow 0} p(t, i, i)=1 \\
p(s+t, i, k)=\sum_{j} p(s, i, j) p(t, j, k) .
\end{gathered}
$$

Let $f$ be a corresponding absolute probability function, that is a function from $(0, \infty) \times I$ into $[0,1]$ satisfying

$$
\sum_{j} f(t, j)=1, \quad f(s+t, j)=\sum_{i} f(s, i) p(t, i, j) .
$$

There is then a Markov process $\{x(t), t>0\}$ with state space $I$ and the specified transition and absolute probability functions. Such a process will be called an $(f, 1)$ process with state space $I$. The transition function, if not otherwise specified, is the given one. All processes in this paper are assumed defined on complete measure spaces.

It has long been known that it is advantageous to find a topological state space $L$ in which there is a countable set identified with $I$, and to use $L$ as a state space, choosing $L$ and the process in such a way that the process sample functions have desirable continuity properties. If $\{x(t), t>0\}$ is a Markov process with absolute probability function $f$ (and transition function $p$ ) with state space a topological space $L \supset I$, and if the process is right continuous with left limits, that is if almost every sample function is right continuous with a left limit on $(0, \infty)$, the process will be called a smooth $(f, 1)$ process with state space $L$, or simply a smooth process with state space $L$ if $f$ is not specified.

(1.2) Conventions on measures. Throughout this paper the transition function will be held fixed and no hypotheses will be imposed on it, other than those in (1.1.1). If $I$ is immersed in a topological space, $p(t, \xi, \cdot)$ and $f(t, \cdot)$ (for $f$ an absolute probability function) are frequently considered measures of Borel subsets of the space, supported by $I$, with $p(t, \xi, j)$ and $f(t, j)$ the measures assigned to the singleton $\{j\}$. The limit measures when $t \rightarrow 0$ may not be supported by $I$.

Convergence of a sequence of measures, $\lim _{n \rightarrow \infty} \mu_{n}=\mu$, always means

$$
\lim _{n \rightarrow \infty} \int \phi d \mu_{n}=\int \phi d \mu
$$

for every bounded continuous $\phi$. The domain of integration is always the whole space unless otherwise specified.

(1.3) Adapted spaces. A space $L \supset I$ will be said to be adapted (to the given transition function) if the following conditions (a), (b) are satisfied; $L$ will be said to be entrance adapted if (a), (b), (c) are satisfied.

(a) $L$ is a Borel subset of a compact metric space in which $I$ is dense.

(b) For every absolute probability function $f$ there is a smooth $(f, 1)$ process with state space $L$. 
(c) For every integer $j, p(\cdot, \cdot, j)$ has a continuous extension to $(0, \infty) \times L$ and

$$
\begin{aligned}
\sum_{j} p(t, \xi, j) & =1, \\
p(s+t, \xi, k) & =\sum_{j} p(s, \xi, j) p(t, j, k), \quad(s, t>0) .
\end{aligned}
$$

Here and in the following $i, j, k$ are integers and $\xi, \eta, \zeta$ are points of the specified topological state space.

(1.4) Definition. If $L$ is an entrance adapted space, a point $\xi$ of $L$ will be called polar if almost no path or path left limit of any smooth process with state space $L$ hits $\xi$. The point $\xi$ will be called a nonbranch point if (a) almost every path of a smooth $(p(\cdot, \xi, \cdot), 1)$ process with state space $L$ has limit $\xi$ at the parameter value 0 , and if this is so $\xi$ will be called regular if (b) almost every path or path left limit of this process hits $\xi$ at arbitrarily small parameter values. (It is easily checked that conditions (a) and (b) depend only on $L$ and $\xi$, not on the particular $(p(\cdot, \xi, \cdot), 1)$ process chosen.)

(1.5) Strong Markov and quasi left continuity properties. Let $\{x(t), t>0\}$ be a smooth $(f, 1)$ process with entrance adapted state space $L$. If $t>0$ let $\mathscr{F}(t)$ be the smallest $\sigma$ algebra with respect to which $x(s)$ is measurable for $s \leqq t$ and which contains all null sets. Then $\mathscr{F}(t+)=\mathscr{F}(t)$ and the strong Markov and quasi left continuity properties are valid in the following forms. (For proofs in practically the present context see [2].) If $\tau$ is a strictly positive stopping time for this family of $\sigma$ algebras, and if $A$ is a Borel subset of $L$,

$$
P\{\tau<\infty, x(\tau+t) \in A \mid \mathscr{F}(t)\}=p(t, x(\tau), A) \quad(t>0)
$$

almost everywhere where $\tau$ is finite. Moreover if $\left\{\tau_{n}, n \geqq 1\right\}$ is an increasing sequence of stopping times with limit $\tau$,

$$
P\left\{\tau<\infty, x(\tau+t) \in A \mid \bigvee_{n} \mathscr{F}\left(\tau_{n}\right)\right\}=p(t, x(\tau-), A) \quad(t>0)
$$

almost everywhere on the set where $\tau_{n}<\tau<\infty$ for all $n$, and $x(\tau-)=x(\tau)$ almost everywhere where simultaneously $\tau_{n}<\tau<\infty$ for all $n$ and the value of $x(\tau-)$ is not a branch point. If $x(0+)$ exists almost surely and if $x(0)$ is defined as this limit, the restriction of positivity of stopping times can be omitted. Since the distribution of $x(\tau+t)$ is supported by $I,(1.5 .1)$ and (1.5.2) are no more general than those equations with $A$ replaced by the singleton $\{j\}$, but the equations are stated with $A$ since in this form they will be extended to the case $t=0$.

\section{Adapted spaces.}

(2.1) Branch points. If $L$ is an entrance adapted space, almost no path of any smooth process with state space $L$ meets a branch point, and the probability is 0 that $x(0+)$ exists and is a branch point. The proof is omitted because [2] contains a proof in an only slightly different context. 
(2.2) Hitting probabilities. If $\{x(t), t>0\}$ is a smooth $(f, 1)$ process with entrance adapted state space $L$ and if $A$ is a Borel subset of $L$ the usual capacity theory argument shows that the probability that a path or path left limit hits $A$ is well defined and is the supremum of this probability for compact subsets of $A$, and that the probability distribution of the hitting time of $A$ (by paths or their left limits) is independent of the choice of smooth $(f, 1)$ process. Furthermore if $\tau_{0}\left[\tau_{1}\right]$ is the infimum of times $t$ at which $x(t)$ or $x(t-)[x(t)]$ is in $A$ then $\tau_{0} \leqq \tau_{1}$ and if $A$ consists only of nonbranch points there is equality almost surely. This fact is familiar when there are no branch points and the proof will only be sketched. It is sufficient to prove that if $0<a<b$ it is almost sure that a path meets $A$ for some parameter value in $[a, b]$ whenever the path or path left limit meets $A$ for some parameter value in that interval. Since the probability of the latter event is the supremum of the corresponding probabilities for compact subsets of $A$ we can suppose in the first place that $A$ is compact. Let $\tau^{\prime}$ be the first point $t$ in $[a, b]$ with $x(t)$ or $x(t-)$ in $A$, or $\tau^{\prime}=\infty$ if there is no such point. It is sufficient to show that $x\left(\tau^{\prime}\right)$ is in $A$ almost surely whenever $\tau^{\prime}<\infty$ and $x\left(\tau^{\prime}-\right)$ is in $A$. If this statement is false there is an increasing sequence $\left\{\tau_{n}, n \geqq 1\right\}$ of stopping times with limit $\tau^{\prime}$ such that the set $\left\{\tau_{n}<\tau^{\prime}\right.$ for all $n, x\left(\tau^{\prime}-\right)$ in $A, x\left(\tau^{\prime}\right)$ not in $\left.A\right\}$ has strictly positive probability, a contradiction to quasi left continuity.

(2.3) Proposition. If $\xi$ is a point of an entrance adapted state $L$ and if $k$ is an integer then $p(\cdot, \xi, k)$ vanishes identically on $(0, \infty)$ if at all.

According to a theorem of Austin [1] the theorem is true if $\xi$ is an integer. It follows from (1.3.1) that for any $\xi$ in $L$ if $p\left(t_{0}, \xi, k\right)=0$ then $p(\cdot, \xi, j)=0$ on $\left(0, t_{0}\right]$ for every $j$ with $p(\cdot, j, k)>0$, and then that $p(\cdot, \xi, k)$ vanishes on $\left(t_{0}, \infty\right)$.

(2.4) Definition. An entrance space is a space $L$ entrance adapted to the given transition function and with the following additional property:

(d) if $\xi$ is in $L$ and if $\{x(t), t>0\}$ is a smooth $(p(\cdot, \xi, \cdot), 1)$ process with state space $L$ then $x(0+)$ exists (and is in $L$ ) almost surely.

(2.5) THEOREM. Let $L$ be an entrance adapted space and let $L_{0}$ be the set of those points $\xi$ of $L$ for which $x(0+)$ exists almost surely when $\{x(t), t>0\}$ is a smooth $(p(\cdot, \xi, \cdot), 1)$ process. Then $L_{0}$ is an entrance space.

Since the limit $x(0+)$ in the definition of $L_{0}$ can be defined as a limit along rational parameter values, $L_{0}$ is a Borel subset of $L$. If $L_{0}$ is not entrance adapted there is an absolute probability function $f$, a smooth $(f, 1)$ process $\{x(t), t>0\}$ with state space $L$, numbers $a, b$ with $0<a<b$, and a compact subset $A$ of $L-L_{0}$ with the property that the probability is strictly positive that an $x$ path or left limit hits $A$ in the parameter interval $[a, b]$. Let $\tau$ be the first such time, or $\tau=b+1$ if there is no such time. Then $x(\tau)$ or $x(\tau-)$ is in $A$ with strictly positive probability. The process $\{x(\tau+t), t \geqq 0\}$ is right continuous, has transition function $p$, and has as initial distribution the distribution of $x(\tau)$. But then $x(\tau+)$ exists almost nowhere 
where $x(\tau)$ is in $A$. Hence $x(\tau)$ is almost never in $A, x(\tau-)$ must be in $A$ with strictly positive probability and it follows that there is an increasing sequence $\left\{\tau_{n}, n \geqq 1\right\}$ of stopping times, with limit $\tau$, such that with strictly positive probability simultaneously $\tau_{n}<\tau$ for all $n$ and the value of $x(\tau-)$ is in $A$. But then according to the strong Markov property in the form (1.5.2) the distribution of the process $\{x(\tau+t)$, $t>0\}$ when $\tau_{n}<\tau$ for all $n$ and $x(\tau-)$ is in $A$ is that of a smooth process with absolute probability function generated by an initial distribution supported by $A$. It follows that with strictly positive probability $x(\tau+)$ does not exist, contrary to fact, and we have finished the proof that $L_{0}$ is entrance adapted. If in this proof $a=0$ and $f=p(\cdot, \xi, \cdot)$ for a point $\xi$ in $L_{0}$, and if $x(0)$ is defined as $x(0+)$, the proof shows that $x(0)$ is almost surely in $L_{0}$. Hence $L_{0}$ is an entrance space.

\section{Entrance spaces.}

(3.1) Hitting times of branch points. We have already noted that almost no path (or its limit at 0 ) of a smooth process with an entrance state space ever meets the set of branch points. There is therefore a discontinuity whenever a nonexceptional path has a branch point as left limit. If $A$ is a compact set of branch points the successive times when a nonexceptional path left limit hits $A$ form a set with no limit point from the right. If $A$ is a singleton the times between hits of $A$ by a nonexceptional path left limit are independent with a common distribution function so there is no limit point of left limit hitting times.

(3.2) Convergence of distributions when $t \rightarrow 0$. If $L$ is an entrance space the existence of $x(0+)$ in Definition 2.4 implies that

$$
\lim _{t \rightarrow 0} p(t, \xi, \cdot)=p(0+, \xi, \cdot)
$$

(convergence of measures) exists for all $\xi$ in $L$, and we define $p(0, \xi, \cdot)$ as the measure on the right. Then $\xi$ is a branch point if and only if $p(0, \xi, \xi)<1$ and the second equation in (1.3.1) can be rewritten to allow $s$ and $t$ to vanish:

$$
p(s+t, \xi, A)=\int_{L} p(t, \eta, A) p(s, \xi, d \eta) \quad(s, t \geqq 0, \xi \in L),
$$

for every Borel subset $A$ of $L$. Similarly (1.5.1) and (1.5.2) are now valid for $t \geqq 0$.

(3.3) THEOREM. If $L$ is an entrance space and if $\{x(t), t>0\}$ is a smooth $(f, 1)$ process with state space $L, x(0+)$ exists almost surely if and only if $f$ has the form

$$
f(t, i)=\int_{L} p(t, \xi, i) p_{0}(d \xi) \quad(t>0)
$$

for some probability measure $p_{0}$. The measure $p_{0}$ can always be chosen to be supported by the set of nonbranch points and if so supported it is determined uniquely by $f$. [In this case (3.3.1) will be called the canonical representation of $f$.] 
It is trivial that if $f$ is of the form (3.3.1) then $x(0+)$ exists almost surely. Conversely if $x(0+)$ exists almost surely,

$$
\lim _{t \rightarrow 0} f(t, \cdot)=f(0+, \cdot)
$$

exists (convergence of measures) and (1.1.2) can be extended to

$$
f(t, A)=\int_{L} p(t, \eta, A) f(0+, d \eta) \quad(t \geqq 0),
$$

valid for every Borel subset $A$ of $L$. Thus $f$ must have the special form (3.3.1). Moreover by $(2.1) f(0+, \cdot)$ is supported by the set of nonbranch points. When $t$ goes to zero in (3.3.1) we find that

$$
f(0+, A)=\int_{L} p(0, \xi, A) p_{0}(d \xi)
$$

and, if $p_{0}$ is supported by the set of nonbranch points this equation reduces to $f(0+, A)=p_{0}(A)$. The proof is complete.

(3.4) Extreme (minimal) absolute probability functions. The set of absolute probability functions is convex. An absolute probability function $f$ is extreme in this set if and only if it is minimal, that is if and only if $f$ is the only absolute probability function dominated by a multiple of $f$.

(3.5) THEOREM. If $\xi$ is a nonbranch point of an entrance space $L, p(\cdot, \xi, \cdot)$ is a minimal absolute probability function.

If $f=p(\cdot, \xi, \cdot)$ is not minimal, we can write $f=\left(f_{1}+f_{2}\right) / 2$, where $f_{1}$ and $f_{2} \neq f_{1}$ are absolute probability functions. Then if $\left\{x_{i}(t), t>0\right\}$ is a smooth $\left(f_{i}, 1\right)$ process with state space $L$ and if $\{x(t), t>0\}$ is a smooth $(f, 1)$ process with state space $L$, the probability that $x(0+)$ exists is the average of the probabilities that $x_{1}(0+)$ and $x_{2}(0+)$ exist. Hence the latter two probabilities are both 1 , and according to Theorem 3.3 the absolute probability functions $f, f_{1}, f_{2}$ have unique canonical representations (3.3.1). Since the measure corresponding to $f$ is supported by $\xi$, the stated uniqueness is impossible, and we have proved that $f$ is minimal.

(3.6) Existence of entrance spaces. It is well known that there is always an entrance space (see for example [2], [4]), and in fact that there is always one which has, besides the properties (a)-(d) of (1.3) and (2.4), the following properties (e) and (f).

(e) $p\left(0, \xi_{1}, \cdot\right)=p\left(0, \xi_{2}, \cdot\right)$ implies that $\xi_{1}=\xi_{2}$. (Of course this implication is trivial if neither point is a branch point.) Moreover if $\lim _{n \rightarrow \infty} p\left(t, \xi_{n}, j\right)=u(t, j)$ exists for $t>0, j$ in $I$, and if $\sum_{j} u(\cdot, j)=1$, then $\lim _{n \rightarrow \infty} \xi_{n}$ exists.

(f) If $f$ is any absolute probability function it has a unique canonical representation (3.3.1) (so that every smooth process with state space $L$ has almost surely a limit at $t=0$ and every minimal $f$ is of the form $p(\cdot, \xi, \cdot)$ for some nonbranch point $\xi)$. 
(3.7) Definition. A canonical entrance space is an entrance space with the properties (e), (f).

Let $L$ be an entrance space and $L^{c}$ a canonical entrance space. Denote by $p^{c}$ the extended transition function for $L^{c}$. If $\xi$ is a nonbranch point of $L$ there is a unique nonbranch point $\xi^{c}$ of $L^{c}$ for which $p^{c}\left(\cdot, \xi^{c}, \cdot\right)=p(\cdot, \xi, \cdot)$. The map from the set $L_{1}$ of nonbranch points of $L$ into its image $L_{1}^{c}$ is continuous because of property (e) of canonical entrance spaces, and is one-to-one. The map therefore makes Borel sets correspond to Borel sets in both directions. Any Borel measure on $L_{1}$ corresponds to a unique Borel measure on $L_{1}^{c}$ and conversely. Thus a canonical representation (3.3.1) on $L$ becomes one on $L^{c}$.

(3.8) Let $L$ and $L^{\prime}$ be entrance spaces, and denote the extensions of the transition function for these spaces by $p$ and $p^{\prime}$ respectively.

THEOREM. Let $L_{0}^{\prime}$ be the set of points $\xi^{\prime}$ in $L^{\prime}$ for which $x(0+)$ exists almost surely whenever $\{x(t), t>0\}$ is a $\left(p^{\prime}\left(\cdot, \xi^{\prime}, \cdot\right), 1\right)$ smooth process with state space $L$ (that is for which $p^{\prime}\left(\cdot, \xi^{\prime}, \cdot\right)$ has a representation (3.3.1) on $\left.L\right)$. Then $L_{0}^{\prime}$ is an entrance space.

In the convergence condition for $\xi^{\prime}$ to be in $L_{0}^{\prime}, x(0+)$ can be defined in terms of approach to 0 along the rationals, and it follows easily that $L_{0}^{\prime}$ is a Borel set. If $L_{0}^{\prime}$ is not entrance adapted there is a smooth process $\left\{x^{\prime}(t), t>0\right\}$ with state space $L^{\prime}$, and a number $\delta>0$ for which a path or path left limit hits $L^{\prime}-L_{0}^{\prime}$ with strictly positive probability in the parameter interval $[\delta, 1 / \delta]$. Choose a compact subset $A$ of $L^{\prime}-L_{0}^{\prime}$ so large that the same assertion is true for $A$ instead of $L^{\prime}-L_{0}^{\prime}$, let $\tau^{\prime}$ be the infimum of times $\geqq \delta$ at which a path or path left limit hits $A$ and let $\tau=\tau^{\prime} \wedge 1 / \delta$. Then $x^{\prime}(\tau)$ or $x^{\prime}(\tau-)$ is in $A$ with strictly positive probability. Define $x(t)=\lim _{s \downarrow t} x^{\prime}(s)$, where the limit is in the $L$ topology and $s$ is restricted to the rationals (so that $x^{\prime}(s)$ is almost surely integer valued simultaneously for all $s$ ). The $x$ process is then a smooth process with state space $L$ and the same absolute probability function as the $x^{\prime}$ process. Let $\left\{\tau_{n}, n \geqq 1\right\}$ be a decreasing sequence with limit $\tau$ of finite rational valued stopping times. By process right continuity if $f_{n}(t, \cdot), f(t, \cdot)$ are respectively the distributions of $x^{\prime}\left(\tau_{n}+t\right), x^{\prime}(\tau+t)$, for $t>0$, there is convergence of measures: $\lim _{n \rightarrow \infty} f_{n}(t, \cdot)=f(t, \cdot)$. Since the distributions involved are all supported by the integers there is pointwise convergence: $\lim _{n \rightarrow \infty} f_{n}(t, j)=f(t, j)$ for all $j$. Now $\tau_{n}$ is a stopping time for the $x$ process so $\tau$ is also, and $x^{\prime}\left(\tau_{n}+t\right)$ has the same distribution as $x\left(\tau_{n}+t\right)$. The preceding reasoning can therefore be applied to the $x$ process and yields the fact that the processes $\left\{x^{\prime}(\tau+t), t>0\right\},\{x(\tau+t), t>0\}$ are smooth $(f, 1)$ processes in their respective state spaces. Since $x^{\prime}(\tau+)$ exists almost surely, $f$ has a canonical representation (3.3.1) based on $L^{\prime}$, with $p_{0}$ the distribution of $x^{\prime}(\tau)$. If $x^{\prime}(\tau)$ is in $A$ with strictly positive probability the distribution of $x^{\prime}(\tau)$ assigns nonzero weight to the set of nonbranch points $\xi^{\prime}$ with $p^{\prime}\left(\cdot, \xi^{\prime}, \cdot\right)$ not of the form (3.3.1) based on $L$. On the other hand the same reasoning applied to the $x$ process shows that $f$ has a canonical representation based on $L$ which assigns zero weight to points yielding such absolute 
probability functions. There are therefore two different representations of $f$ when referred back to the representations based on a canonical entrance space. We have arrived at a contradiction and thereby deduce that $x^{\prime}(\tau)$ is almost never in $A$. Hence $x^{\prime}(\tau-)$ is in $A$ with strictly positive probability, and it follows that there is an increasing sequence $\left\{\tau_{n}^{\prime}, n \geqq 1\right\}$ of stopping times with limit $\tau$ such that with strictly positive probability: $\tau_{n}^{\prime}<\tau$ for all $n ; \tau_{n}^{\prime}$ is rational valued; the value of $x(\tau-)$ is in $A$. According to the strong Markov property in the form (1.5.2) the distribution of the process $\left\{x^{\prime}(\tau+t), t>0\right\}$ where $\tau_{n}^{\prime}<\tau$ for all $n$ and $x^{\prime}(\tau-)$ is in $A$ is that of a smooth process with state space $L^{\prime}$ and absolute probability function generated by an initial distribution which assigns strictly positive weight to $A$. Repeating the reasoning used already, the distribution of the process $\{x(\tau+t)$, $t>0\}$ when $\tau_{n}^{\prime}<\tau$ for all $n$ and $x^{\prime}(\tau-)$ is in $A$ is that of a smooth process with state space $L$, the absolute probability function just described, and this fact contradicts the fact that $x(\tau+)$ exists almost surely. We have thus proved that $L^{\prime}$ is entrance adapted. If $\xi^{\prime}$ is in $L_{0}^{\prime}$ almost every path of a smooth $\left(p^{\prime}\left(\cdot, \xi^{\prime}, \cdot\right), 1\right)$ process $\left\{y^{\prime}(t), t>0\right\}$ with state space $L^{\prime}$ has limit $\xi^{\prime}$ at $t=0$ if $\xi^{\prime}$ is not a branch point. If $\xi^{\prime}$ is a branch point the same proof as that given above showing that $x^{\prime}(\tau)$ is almost never in $A$ shows here that $y^{\prime}(0+)$ is almost never in $A$. Hence $L_{0}^{\prime}$ is an entrance space, as was to be proved.

(3.9) THEOREM. Let $L, L^{\prime}$ be as in the preceding theorem.

(a) If $\xi^{\prime}$ is a point of $L^{\prime}$ which is neither polar nor a branch point, there is a unique nonbranch point $\xi$ of $L$ with $p(\cdot, \xi, \cdot)=p^{\prime}\left(\cdot, \xi^{\prime}, \cdot\right)$.

(b) If $A^{\prime}$ is a Borel set of nonbranch nonpolar points of $L^{\prime}$ and if $A$ is the corresponding subset of $L$ furnished by (a), $A$ is a Borel set and, for every absolute probability function $f$ the hitting time distribution of $A^{\prime}$ for a smooth $(f, 1)$ process with state space $L^{\prime}$ is the same as the hitting time distribution of $A$ for a smooth $(f, 1)$ process with state space L. In particular $\xi$ in (a) is nonpolar, and is regular if $\xi^{\prime}$ is a regular point of $L^{\prime}$.

If $\xi^{\prime}$ is not polar, $\xi^{\prime}$ cannot be in the set $L^{\prime}-L_{0}^{\prime}$ of Theorem 3.8 , because this set is not hit by smooth processes or their left limits. Hence the absolute probability function $p^{\prime}\left(\cdot, \xi^{\prime}, \cdot\right)$ has a canonical representation (3.3.1) based on $L$. If $\xi^{\prime}$ is not a branch point, $p^{\prime}\left(\cdot, \xi^{\prime}, \cdot\right)$ is minimal, so the measure $p_{0}$ must be supported by a singleton $\{\xi\}$. Thus (a) is true. To prove (b) note that the nonbranch points of $L$ and $L^{\prime}$ are each in a one-to-one Borel set preserving correspondence with subsets of the nonbranch points of a canonical entrance space. Hence in (b) if $A^{\prime}$ is a Borel set, $A$ is also a Borel set. Let $\left\{x^{\prime}(t), t>0\right\}$ be a smooth $(f, 1)$ process with state space $L^{\prime}$ and define $x(t)=\lim _{s \downarrow t} x^{\prime}(s)$, where the limit is in the $L$ topology and $s$ is restricted to be rational, so that $x^{\prime}(s)$ can be taken as integer valued. Let $x^{\prime}(t, \omega)$ be the value of $x^{\prime}(t)$ at the point $\omega$ of the measure space on which the $x^{\prime}$ process is defined, and we write $x(t, \omega)$ for the value of $x(t)$ at $\omega$. Deleting a null $\omega$ set, the $x$ process is a smooth $(f, 1)$ process with state space $L$. Now almost no path of a 
smooth process hits a branch point. Thus deleting a null $\omega$ set if necessary we can assume that $x^{\prime}(t, \omega)$ and $x(t, \omega)$ are not branch points, and we prove that if $x^{\prime}(t, \omega)$ is not polar its image under the correspondence set up in (a) is precisely $x(t, \omega)$. This assertion is trivially true when $t$ is restricted to the rationals because integers in $L$ and $L^{\prime}$ correspond to each other. Therefore (restricting $s$ to the rationals)

$$
\begin{aligned}
p^{\prime}\left(t, x^{\prime}(t, \omega), j\right) & =\lim _{s \downarrow t} p^{\prime}\left(s, x^{\prime}(s, \omega), j\right) \\
& =\lim _{s \downarrow t} p(s, x(s, \omega), j) \\
& =p(t, x(t, \omega), j),
\end{aligned}
$$

and this equality identifies $x(t, \omega)$ as the image of $x^{\prime}(t, \omega)$. Now in proving (b) we can, according to $(2.2)$, use any smooth $(f, 1)$ processes we wish. If we use the pair we have been considering, it is clear that the hitting time of $A^{\prime}$ is almost surely less than or equal to that of $A$. On the other hand if in the present context, after deleting the null sets as described, if $x\left(t_{0}, \omega\right)$ is in $A$, the preceding reasoning carried through in the reverse direction shows that $x\left(t_{0}, \omega\right)$ is the image of $x^{\prime}\left(t_{0}, \omega\right)$ in the correspondence set up in (a). Hence the hitting time of $A^{\prime}$ is greater than or equal to that of $A$, and the theorem is now completely proved. Incidentally we have proved that (deleting appropriate $\omega$ null sets) the paths of the $x^{\prime}$ and $x$ processes are images of each other, if observed only when the paths are at nonbranch nonpolar points.

\section{Exit spaces.}

(4.1) Generalities. The conditions on an entrance space involve only the given transition function $p$. Unfortunately the transition function of a Markov process reversed in time depends on the absolute probability function. We shall choose an absolute probability function, reverse the corresponding process starting from a random time to get a stationary reverse transition function $p^{*}$ and define an exit space for $p$ as an entrance space for $p^{*}$.

(4.2) $A$ dual transition function. Let $\left\{a_{n}, n \in I\right\}$ be strictly positive numbers with sum 1. Define

$$
\begin{gathered}
f^{\circ}(t, j)=\sum_{i} a_{i} p(t, i, j), \\
h(j)=\int_{0}^{\infty} e^{-s} f^{\circ}(s, j) d s ; \quad p_{h}(t, i, j)=p(t, i, j) / h(j) .
\end{gathered}
$$

Then the function $h$ is strictly positive.

If $S$ is any space, $S^{\Delta}$ will denote that space with a new point $\Delta$ adjoined to it. If $S$ is topological, $S^{\Delta}$ is also and the open subsets of $S^{\Delta}$ are the open subsets of $S$ with or without $\Delta$, so that $\Delta$ is an isolated point of $S^{\Delta}$.

Define a transition function for the state space $I^{\Delta}$ by

$$
\begin{gathered}
p^{*}(t, i, j)=e^{-t} p_{h}(t, j, i) h(j), \quad p^{*}(t, i, \Delta)=\int_{0}^{t} e^{-s} f^{0}(s, i) d s / h(i), \\
p^{*}(t, \Delta, \Delta)=1 .
\end{gathered}
$$


This transition function satisfies (1.1.1) on $I^{\Delta}$ and will be called the dual of the transition function $p$. This dual depends on the choice of $a_{.}$, which will be fixed throughout this paper, except in (9.5).

(4.2.3) If $M$ is a topological space and if $M^{\Delta}$ is an entrance space for $p^{*}, M$ will be called an exit space for $p$. For consistency one can extend the transition function $p$ by defining

$$
\begin{aligned}
p^{\Delta}(t, i, j) & =p(t, i, j), \\
p^{\Delta}(t, i, \Delta) & =0, \quad(t>0 ; i, j \text { in } I) . \\
p^{\Delta}(t, \Delta, \Delta) & =1,
\end{aligned}
$$

If $L$ is an entrance space for $p$ then $L^{\Delta}$ is one for $p^{\Delta}$. Conversely $\Delta$ is an isolated point of any entrance space for $p^{\Delta}$ and the space less this point is an entrance space for $p$. If $f$ is an absolute probability function for $p$ and if $f^{\Delta}$ is $f$ extended by setting $f(\cdot, \Delta)=0$, the extended function is an absolute probability function for $p^{\Delta}$. A smooth $(f, 1)$ process with state space $L$ becomes a smooth process on $L^{\Delta}$ for $p^{\Delta}$ under the convention that any path which reaches $\Delta$ stays there.

If $L^{*}$ is an exit space for $p$, the functions $p^{*}(\cdot, \cdot, j)$ and $p^{*}(\cdot, \cdot, \Delta)$ have continuous extensions to $(0, \infty) \times L^{*}$. Then $p_{h}(\cdot, j, \cdot)$ has a continuous extension to $(0, \infty) \times L^{*}$. In the following, such notation as $p_{h}$ will always refer to the extended function. The second equation of (1.3.1) when applied to $p^{*}$ implies that for $\xi^{*}$ in $L^{*}$

$$
p_{h}\left(s+t, k, \xi^{*}\right)=\sum_{j} p(s, k, j) p_{h}\left(t, j, \xi^{*}\right)
$$

(4.3) Probabilistic interpretations. Using $f^{0}$ (defined in (4.2.1)) let $\left\{x_{0}(t), t>0\right\}$ be an $\left(f^{\circ}, 1\right)$ process with state space $I$. Let $Z$ be a positive random variable, independent of this process, with distribution density $s \leadsto e^{-s}$ on $[0, \infty)$. If $x_{0}(t)$ is defined as $\Delta$ for $t \leqq 0$, define the process $\left\{x_{0}^{*}(t), t \geqq 0\right\}$ by $x_{0}^{*}(t)=x_{0}(Z-t)$. This process is a Markov process with transition function $p^{*}$. The distribution of each random variable of the process is supported by $I^{\Delta}$ and the absolute probability function of the process is given by

$$
f^{0 *}(t, i)=e^{-t} h(i), \quad f^{0 *}(t, \Delta)=1-e^{-t} .
$$

In particular, if $L$ is an adapted space for $p$, in the sense of (1.3), the $x_{0}$ process can be taken to be smooth with state space $L$ and the process $\left\{x_{0}^{*}(t+), t>0\right\}$ will then be a smooth process for $p^{*}$.

\section{Entrance exit spaces.}

(5.1) Proposition. If $L$ is adapted to $p$ then $L^{\Delta}$ is adapted to $p^{*}$.

According to (4.3) if $L$ is adapted to $p$, the process

$$
\left\{\lim _{s \downarrow t} x_{0}^{*}(s), t>0\right\} \quad \text { (s rational) }
$$


(limit in the $L^{\Delta}$ topology) has state space $L^{\Delta}$ and is a right continuous Markov process with left limits, absolute probability function $f^{0 *}$, transition function $p^{*}$. The absolute probability function $f^{0 *}$ is generated by the initial distribution assigning probability $h(j)$ to $j$. Hence there is a smooth process with this state space and transition function for any absolute probability function generated by an initial distribution supported by $I^{\Delta}$. If $f^{*}$ is an arbitrary absolute probability function for $p^{*}$, let $\{y(t), t>0\}$ be a Markov process with state space $I^{\Delta}$, transition function $p^{*}$, absolute probability function $f^{*}$. According to what we have just proved, if $c>0$ the process

$$
\left\{\lim _{s \downarrow t} y(s), t>c\right\} \quad \text { (s rational) }
$$

(limit in the $L^{\Delta}$ topology), which has absolute probability function generated by the initial distribution $f^{*}(c, \cdot)$, supported by $I^{\Delta}$, is right continuous with left limits. Since $c$ is arbitrary, $L^{\Delta}$ is adapted to $p^{*}$.

(5.2) Proposition. If $L^{* \Delta}$ is adapted to $p^{*}$ then $L^{*}$ is adapted to $p$.

If $L^{* \Delta}$ is adapted to $p^{*}$, and if the limit in (5.1.1) is taken in the $L^{*}$ topology, the process so defined is a right continuous process with left limits, state space $L^{* \Delta}$. That is, $x_{0}(t)$ can be modified for $t<Z$ to be $L^{*}$ valued, left continuous with right limits (or, if preferred, right continuous with left limits, by setting $x(t)=\lim _{s \downarrow t} x_{0}(s)$, with $s$ rational, limit in the $L^{*}$ topology). Since $Z$ is independent of the $x_{0}$ process, $x(t)$ exists as the indicated limit almost surely simultaneously for all $t>0$ and the $x$ process is a smooth $\left(f^{0}, 1\right)$ process with state space $L^{*}$. The fact that there is a smooth $(f, 1)$ process, for an arbitrary $f$, with state space $L^{*}$ is proved for $f$ generated by an initial distribution on $I$ and then for arbitrary $f$ just as in the proof of Proposition 5.1.

(5.3) THEOREM. There is a space which is both an entrance and an exit space for the given transition function.

Let $K_{0}$ and $K_{0}^{*}$ be respectively entrance and exit spaces for $p$. Then $K_{0}\left[K_{0}^{*}\right]$ is a Borel subset of a compact metric space $K\left[K^{*}\right]$ with metric $\rho\left[\rho^{*}\right]$. Let $\hat{K}$ be the completion of $I$ in the metric $\rho+\rho^{*}$. Then $\hat{K}$ is a compact metric space in which $I$ is dense and there is a unique continuous map $\alpha\left[\alpha^{*}\right]$ from $\hat{K}$ into $K\left[K^{*}\right]$ leaving $I$ invariant. Define

$$
\begin{aligned}
\hat{K}_{0} & =\alpha^{-1}\left(K_{0}\right) \cap \alpha^{*-1}\left(K_{0}^{*}\right), \\
\hat{p}(t, \hat{\xi}, A) & =p(t, \alpha(\hat{\xi}), A), \\
\hat{p}^{*}(t, \hat{\xi}, j) & =p^{*}\left(t, \alpha^{*}(\hat{\xi}), j\right),
\end{aligned}
$$

so that

$$
\hat{p}(t, i, j)=p(t, i, j), \quad \hat{p}_{h}(t, i, j)=p_{h}(t, i, j), \quad \hat{p}^{*}(t, i, j)=p^{*}(t, i, j) .
$$


Adjoin a point $\Delta$ to $\hat{K}_{0}$, making $\Delta$ an isolated point of the enlarged space $\hat{K}_{0}^{\Delta}$. Extend $\hat{p}$ to $\hat{p}^{\Delta}$ following (4.2) and define

$$
\hat{p}^{*}(t, \Delta, \Delta)=1, \quad \hat{p}^{*}(t, \hat{\xi}, \Delta)=p^{*}\left(t, \alpha^{*}(\hat{\xi}), \Delta\right) .
$$

For each integer $j$, the functions $\hat{p}(\cdot, \cdot, j)$ and $\hat{p}^{*}(\cdot, \cdot, j)$ are continuous on $(0, \infty)$ $\times \hat{R}_{0}$, and (1.3.1) is satisfied by $\hat{p}$ and $\hat{p}^{*}$. We shall write of spaces adapted to $\hat{p}$ or $\hat{p}^{*}$. Any subset of $\hat{K}_{0}^{\Delta}$ which is adapted to $\hat{p}$ or $\hat{p}^{*}$ is entrance adapted to it. The space $\hat{K}_{0}^{\Delta}$ is adapted to $\hat{p}^{\Delta}$ (equivalently to $\hat{p}$ ) because if $\{x(t), t>0\}$ is an $(f, 1)$ process with state space $I$ we have seen in proving Propositions 5.1 and 5.2 that the restriction to the rationals of almost every $x$ process sample function has right and left limits in $K_{0}\left[K_{0}^{*}\right]$ at every $t>0$, in the $K\left[K^{*}\right]$ topology, therefore also in the $\hat{K}$ topology, and the limits are in $\hat{K}_{0}$. The right limit defines a smooth $(f, 1)$ process with state space $\hat{K}_{0}$. According to Proposition $5.1, \hat{K}_{0}^{\Delta}$ is entrance adapted to $\hat{p}^{*}$ as well as to $\hat{p}^{\Delta}$. By Theorem 2.5 a certain Borel subset $L_{1}$ of $\hat{K}_{0}^{\Delta}$ is an entrance space for $\hat{p}$ and therefore (Proposition 5.1) $L_{1}^{\Delta}$ is entrance adapted to $\hat{p}^{*}$. Then (Proposition 5.2) a certain Borel subset $L_{2}^{\Delta}$ of $L_{1}^{\Delta}$ is an entrance space for $\hat{p}^{*}$ and therefore (Proposition 5.2) $L_{2}$ is an enfrance adapted space for $\hat{p}$, and so on, continuing the alternations. Clearly if $L=\bigcap_{n} L_{n}, L$ is both an entrance space and an exit space for $\hat{p}$, that is, for the given transition function.

(5.4) Common branch points. If $L$ is both an entrance and an exit space, almost no path (including the limit at 0 ) or path left limit of a smooth process with state space $L$ meets the set of points which are branch points both for $p$ and its dual, because paths of a smooth process for a transition function do not meet branch points for that transition function. Hence if the set of points which are branch points both for $p$ and its dual are removed from $L$, the remainder is still an entrance and an exit space. Thus there is always an entrance exit space in the sense of the following definition.

Definition. A space $L$ will be called an entrance exit space if it is both an entrance and an exit space and if no point is a branch point both for the given transition function and its dual.

We observe that if in (5.3) $K_{0}$ and $K_{0}^{*}$ had been chosen to be canonical, the final space $L$ obtained would have had the property that a point of $L$ is determined uniquely by the pair $\left(\hat{p}(\cdot, \hat{\xi}, \cdot), \hat{p}^{*}(\cdot, \hat{\xi}, \cdot)\right)$ that is by the pair $\left(\hat{p}(\cdot, \hat{\xi}, \cdot), \hat{p}_{h}(\cdot, \cdot, \hat{\xi})\right)$ of functions on $(0, \infty) \times I$, and that $\lim _{n \rightarrow \infty} \hat{\xi}_{n}=\hat{\xi}$ if and only if $\lim _{n \rightarrow \infty} \hat{p}\left(\cdot, \hat{\xi}_{n}, \cdot\right)$ $=\hat{p}(\cdot, \hat{\xi}, \cdot)$ and $\lim _{n \rightarrow \infty} \hat{p}_{h}\left(\cdot, \cdot, \hat{\xi}_{n}\right)=\hat{p}_{h}(\cdot, \cdot, \hat{\xi})$. Thus there is always an entrance exit space (a 'canonical' one) satisfying these conditions. We shall not need such spaces in this paper.

(5.5) Proposition. Let $L$ be an entrance exit space. Then a point is a regular point for $p$ if and only if it is regular for $p^{*}$.

Suppose that $\xi$ is regular for $p^{*}$. Then $\xi$ cannot be a branch point for $p$ or an irregular nonbranch point for $p$ because in either case the parameter values where 
$\xi$ is hit by almost any path or path left limit of a smooth process with state space $L$ have no finite limit point (see (3.1)). The converse is proved in the same way.

\section{Space time excessive functions on an entrance space.}

(6.1) Definitions. Throughout this section, $L$ is an entrance space (for $p$ ). If $\alpha>0$, a function $g$ from $(0, \infty) \times L[(0, \infty) \times I]$ into $[0, \infty]$ will be called space time $\alpha$ supermedian for $L[I]$ if

$$
e^{-\alpha s} \sum_{j} p(s, \xi, j) g(t-s, j) \leqq g(t, \xi) \quad(0<s<t)
$$

for $\xi$ in $L[I]$. With either domain the left side of (6.1.1) increases as $s$ decreases, and we denote the limit at 0 by $g_{0}$. The function $g_{0}$ is space time $\alpha$ supermedian. For either domain the minimum of two space time $\alpha$ supermedian functions is also space time $\alpha$ supermedian. In particular if $g$ is such a function so is $g \wedge c$ for every positive constant $c$.

With either domain, if $g=g_{0}$ the function $g$ will be called $\alpha$ space time excessive. The usual argument shows that $g_{0}$ is always space time $\alpha$ excessive. If $g$ is space time $\alpha$ supermedian [excessive] and does not actually depend on its first argument, $g$ is called $\alpha$ supermedian [excessive]. Trivially, if $g$ is space time $\alpha$ supermedian or space time $\alpha$ excessive for $L$ the restriction to $(0, \infty) \times I$ has the same property for $I$.

(6.2) THEOREM. If $g$ is space time $\alpha$ supermedian for $I, g(t-, i)$ exists for every $t>0$ and

$$
g_{0}(t, i)=g(t-, i) \leqq g(t, i) \leqq \liminf _{s \downarrow t} g(s, i) .
$$

If $g$ is space time a supermedian for $I$, it is space time $\alpha$ excessive if and only if it is left continuous in the first argument.

If $g$ is bounded, when $s \rightarrow 0$ in (6.1.1) it follows that $g(t-, i)$ exists and that $g_{0}(t, i)=g(t-, i)$. If this result is applied to $g \wedge c$ when $g$ is not bounded, we find that $g(t-, i)$ exists and

$$
e^{-\alpha s} \sum_{j} p(s, i, j)[g(t-s, j) \wedge c] \leqq g(t-, i) \wedge c \leqq g_{0}(t, i)
$$

When $c \rightarrow \infty$ and then $s \rightarrow 0$ we obtain

$$
g_{0}(t, i) \leqq g(t-, i) \leqq g_{0}(t, i) .
$$

Furthermore $g \geqq g_{0}$ and

$$
\liminf _{\delta \downarrow 0} g(t+\delta, i) \geqq \liminf _{\delta \downarrow 0} e^{-\alpha \delta} p(\delta, i, i) g(t, i)=g(t, i) .
$$

Thus (6.2.1) is true. The last assertion of the theorem is now trivial.

(6.3) Corollary. If $g$ is space time $\alpha$ excessive on $L, g$ is lower semicontinuous on $(0, \infty) \times L$. 
In fact for fixed $s$ the summands in (6.1.1) are lower semicontinuous for $t>s$, $\xi$ in $L$.

The study of space time $\alpha$ supermedian functions is parallel to that of $\alpha$ supermedian functions in [2] except for Theorem 9.3b and Theorem 9.4 of that paper which are not extended because the proofs in [2] are wrong and the truth of the theorems doubtful. Let $L_{b}$ be the set of branch points of the entrance space $L$.

(6.4) THEOREM. If $g$ is space time $\alpha$ supermedian for $L$ and is lower semicontinuous then $g=g_{0}$ on $(0, \infty) \times\left(L-L_{b}\right)$.

In fact by definition of $g_{0}$

$$
g_{0}(t, \xi)=\lim _{s \rightarrow 0} e^{-\alpha s} \int g(t-s, \zeta) p(s, \xi, d \zeta) \geqq \int g(t, \zeta) p(0, \xi, d \zeta),
$$

if $g$ is lower semicontinuous. This inequality implies that when $\xi$ is not a branch point $g_{0}(t, \xi) \geqq g(t, \xi)$, and since the reverse inequality is true there is equality.

(6.5) THEOREM. If $g_{1}$ and $g_{2}$ are space time $\alpha$ excessive for $I, g_{1} \wedge g_{2}$ is also. If $g_{1}$ and $g_{2}$ are space time $\alpha$ excessive for $L, g_{1} \wedge g_{2}$ is $\alpha$ supermedian and coincides on $(0, \infty) \times\left(L-L_{b}\right)$ with the space time $\alpha$ supermedian function $\left(g_{1} \wedge g_{2}\right)_{0}$.

Since the minimum is supermedian, the assertions follow from (6.2), (6.3), (6.4).

(6.6) THEOREM. Let $g$ be a space time $\alpha$ excessive function for $L$.

(a) For every $\xi$ in $L$,

$$
g(t, \xi)=\int g(t, \eta) p(0, \xi, d \eta)
$$

(b) If $\xi \in L-\left(L_{b} \cup I\right)$ then $g(t, \xi)=\lim \inf g(s, i)$ when $i \rightarrow \xi(i$ in $I), s \rightarrow t$.

The truth of (a) follows from

$$
\begin{aligned}
\int g(t, \eta) p(0, \xi, d \eta) & =\lim _{s \rightarrow 0} \int p(0, \xi, d \eta) \int e^{-\alpha s} g(t-s, \zeta) p(s, \xi, d \zeta) \\
& =\lim _{s \rightarrow 0} \int g(t-s, \zeta) p(s, \xi, d \zeta)=g(t, \xi) .
\end{aligned}
$$

To prove (b) let $m(A, \delta)$ be the infimum of $g(t, j)$ for $t<\delta$ and $j$ in the set of integers in an open subset $A$ of $L$ containing $\xi$. Then

$$
\begin{aligned}
g(t, \xi) & =\lim _{s \rightarrow 0} \sum_{j} p(s, \xi, j) g(t-s, j) \\
& \geqq m(A, \delta) \liminf _{s \rightarrow 0} p(s, \xi, A) \\
& =m(A, \delta),
\end{aligned}
$$

and this inequality combined with the lower semicontinuity of $g$ implies the truth of (b). 
(6.7) Extension of space time $\alpha$ excessive functions for $I$. If $g$ is a space time $\alpha$ excessive function for $I$ and if $g^{\prime}(t, \cdot)$ is defined as $g(t, \cdot)$ on $I$ and $+\infty$ otherwise on $L, g^{\prime}$ is space time $\alpha$ supermedian for $L$ and $g_{0}^{\prime}$ is therefore a function which is a space time $\alpha$ excessive (for $L$ ) extension of $g$. The very definition of a space time $\alpha$ excessive function for $L$ shows that such a function is determined by its values on $(0, \infty) \times I$. Thus the extension we have obtained is the only possible one.

(6.8) THEOREM. If $g$ is a space time $\alpha$ excessive function for $L$, if $a>0$, and if $\{x(t), t>0\}$ is a smooth $(f, 1)$ process with state space $L$, the process

$$
\left\{e^{-\alpha t} g[a-t, x(t)], 0<t<a\right\}
$$

is right continuous with left limits. If $f$ has the special form (3.3.1) there is the almost sure limit $g[a, x(0+)]$ at $t=0$.

If $0<\delta<t$, define $\hat{g}_{\delta}$ by

$$
\begin{aligned}
\hat{g}_{\delta}(t, \xi) & =\sum_{j}(1 / \delta) \int_{0}^{\delta} e^{-\alpha s} p(s, \xi, j) g(t-s, j) d s \\
& =\sum_{j}(1 / \delta) \int_{t-\delta}^{t} e^{-\alpha(t-s)} p(t-s, \xi, j) g(s, j) d s .
\end{aligned}
$$

Then $\hat{g}_{\delta}$ increases to $g$ when $\delta \downarrow 0$. Moreover the process

$$
\left\{e^{-\alpha t} \hat{g}_{\delta}[a-t, x(t)], \delta<t<a\right\}
$$

is a supermartingale if the expectations of the random variables are finite. Now if $g$ is bounded by $c$ the series in (6.8.2) is dominated term by term for $(t, \xi)$ in $(\delta, \infty) \times L=A$ by the series

$$
\sum_{j}(c / \delta) \int_{0}^{\delta} p(s, \xi, j) d s
$$

This series, a series of continuous functions on $L$ with continuous sum $c$, converges uniformly on every compact subset of $L$, by Dini's theorem. Then the series (6.8.2) converges uniformly for $(t, \xi)$ in any compact subset of $A$ and it follows that $\hat{g}_{\delta}$ is continuous on $A$. Thus if $g$ is bounded the martingale (6.8.3) is right continuous with left limits. In the bounded case we have now proved that the process (6.8.1) is the limit of an increasing sequence of right continuous supermartingales (all relative to the same family of $\sigma$ algebras) and as such is itself right continuous with left limits, by a theorem of Meyer. If $g$ is not bounded, the process (6.8.1) with $g$ replaced by $(g \wedge c)_{0}$ has the asserted continuity properties and since almost no sample path of the $x$ process hits a branch point the subscript can be omitted, according to Theorem 6.4. Hence the first assertion of Theorem 6.8 is true. In proving the second assertion we can suppose that $g$ is bounded and we define $x(0)=x(0+)$. Since the process $(6.8 .1)$ is a bounded right continuous supermartingale there is a limit at 0 , and by lower semicontinuity

$$
g[a, x(0)] \leqq \lim _{t \rightarrow 0} g[a-t, x(t)] .
$$


On the other hand the process (6.8.1) supplemented by the random variable $g[a, x(0)]$ at $t=0$ is a supermartingale and applying the supermartingale inequality and Fatou's lemma,

$$
E\{g[a, x(0)]\} \geqq E\left\{\lim _{t \rightarrow 0} g[a-t, x(t)]\right\} .
$$

There is therefore equality in (6.8.4), as was to be proved.

7. Exit laws. Throughout this section $L$ is an entrance space.

(7.1) Definition. A function $g$ from $(0, \infty) \times L[(0, \infty) \times I]$ into $[0, \infty]$ will be called an exit law for $L[I]$ if

$$
\sum_{j} p(s, \xi, j) g(t, j)=g(s+t, \xi) \quad(s, t>0)
$$

for $\xi$ in $L[I]$.

If $g$ is a finite valued exit law for $I$, the functional equation (7.1.1) forces $g(\cdot, i)$ to be continuous, with a continuous extension (finite) to $[0, \infty)$, by a theorem of Chung [1]. The restriction to $(0, \infty) \times I$ of an exit law for $L$ is an exit law for $I$. Conversely if $g$ is an exit law for $I$, the left side of (7.1.1) depends only on $s+t$ for $\xi$ in $L$, and defines an extension of $g$ to an exit law for $L$.

If $g$ is an exit law for $L, g$ is a space time $\alpha$ excessive function for $L$, for all $\alpha \geqq 0$. Then $g$ is lower semicontinuous and, from (7.1.1), if $g$ is bounded $g$ is even continuous.

(7.2) THEOREM. If $g$ is an exit law for $I$, if $\alpha \geqq 0$, and if $u$ is defined by

$$
u(i)=\int_{0}^{\infty} e^{-\alpha s} g(s, i) d s
$$

then $u$ is $\alpha$ excessive. For any $i$ with $u(i)$ finite,

$$
\lim _{t \rightarrow \infty} e^{-\alpha t} \sum_{j} p(t, i, j) u(j)=0
$$

and $g(\cdot, i)$ is uniquely determined by $u$. Conversely if $\alpha \geqq 0$ and if $u$ is a finite valued $\alpha$ excessive function satisfying (7.2.2) for all $i$, there is a unique exit law $g$ for which (7.2.1) is true.

Neveu [4] proved this theorem (by a different method) for a slightly more restrictive class of exit laws. Equation (7.1.1) implies that

$$
e^{-\alpha t} \sum_{j} p(t, i, j) u(j)=\int_{t}^{\infty} e^{-\alpha s} g(s, i) d s
$$

which in turn implies that $u$ is $\alpha$ excessive and that (7.2.2) is true if $u(i)$ is finite. Moreover if $u(i)$ is finite, $u$ determines the integral in (7.2.3) and thereby determines $g(\cdot, i)$. Conversely if $\alpha \geqq 0$ and if $u$ is an $\alpha$ excessive finite valued function, the 
function $g_{i}$ defined by

$$
g_{i}(t)=u(i)-e^{-\alpha t} \sum_{j} p(t, i, j) u(j)
$$

is monotone increasing on $(0, \infty)$, from 0 to $u(i)$ if (7.2.2) is satisfied, and we can write

$$
u(i)=\int_{0}^{\infty} e^{-\alpha s} d Q(s, i), \quad Q(t, i)=\int_{0}^{t} e^{\alpha s} d g_{i}(s)
$$

The function $Q(\cdot, i)$ is monotone increasing and

$$
\sum_{i} p(r, k, i) Q(t, i)=Q(t+r, k)-Q(r, k),
$$

from which functional equation it follows [1] that $Q(\cdot, i)$ has a continuous derivative defining an exit law. Thus (7.2.5) yields the desired converse.

(7.3) Generalization. If the condition (7.2.2) is dropped from the hypotheses of the converse in Theorem 7.2 and if $u_{\infty}(i)$ is defined as the limit in (7.2.2), the conclusion becomes

$$
u(i)=\int_{0}^{\infty} e^{-\alpha t} g(t, i) d t+u_{\infty}(i)
$$

where

$$
u_{\infty}(i)=e^{-\alpha t} \sum_{j} p(t, i, j) u_{\infty}(j)
$$

The representation (7.3.1) is a version in the present context of the Riesz decomposition of a positive superharmonic function into the sum of a potential and a harmonic function.

(7.4) Extension of (7.2.1) to $L$. Let $u$ be an $\alpha$ excessive function for $I$, finite valued and represented in terms of the exit law $g$ by (7.2.1). We know that $u$ has a unique $\alpha$ excessive extension to $L$, also denoted by $u$, and $g$ has an extension to an exit law for $L$, also denoted by $g$. Moreover from (7.1.1),

$$
\sum_{j} e^{-\alpha s} p(s, \xi, j) u(j)=\int_{s}^{\infty} e^{-t \alpha} g(t, \xi) d t,
$$

and when $s$ goes to zero in this equation we obtain

$$
u(\xi)=\int_{0}^{\infty} e^{-\alpha t} g(t, \xi) d t,
$$

extending (7.2.1). If $u$ is any $\alpha$ excessive function on $L$ which is finite valued on $I$, this argument shows that there is a unique exit law $g$ for $L$ giving the representation (7.4.2). Conversely direct calculation shows that if $g$ is an arbitrary exit law for $L$, (7.4.2) defines an $\alpha$ excessive function $u$ on $L$.

(7.5) Exit laws on processes. From Theorem 6.8 it is clear that if $\{x(t), t>0\}$ is a smooth $(f, 1)$ process with $f$ of the special form (3.3.1), with state space $L$, if $g$ 
is an exit law on $L$, and if $a>0$, the process $\{g[a-t, x(t)], 0<t<a\}$ is right continuous with left limits, and right limit $g[a, x(0+)]$ at $t=0$. This process is a martingale if

$$
E\{g[a-t, x(t)]\}=\sum_{j} g(a-t, j) f(t, j)<\infty .
$$

(7.6) TheOREM. If $g$ is an exit law for I or $L$ then for each $\xi$ the function $g(\cdot, \xi)$ vanishes identically if at all. If $g$ is an exit law for $L$ and if $A$ is the set of zeros of $g(t, \cdot)$, almost no path of a smooth process with state space $L$ which ever hits $A$ or has limit in $A$ at $t=0$ or has left limit in a nonbranch point of $A$ ever hits $L-A$ or has left limit in $L-A$ thereafter. If $g$ is continuous, in particular if $g$ is bounded, the word 'nonbranch' can be deleted.

The first assertion of the theorem reduces to Proposition 2.3 when $g=p(\cdot, \cdot, k)$ and the method of proof of that proposition is applicable. (We are dealing with entrance spaces instead of entrance adapted spaces here only to avoid pointless verbiage.) The second assertion of the theorem implies for this choice of $g$ that if $p(\cdot, \xi, k)=0$ almost no path of a smooth process with state space $L$ which hits $\xi$ or has limit $\xi$ at $t=0$ or has left limit $\xi$ ever hits $k$ thereafter. To prove the second assertion of the theorem suppose first that $g$ is bounded and continuous and consider the martingale defined in (7.5), assuming, to avoid trivial complications, that $f$ has the special form (3.3.1) and that $x(0)$ is defined as $x(0+)$. Let $\tau_{1}$ be the infimum of times $t$ when $x(t)$ or $x(t-)$ is in a compact subset $A_{1}$ of $A$ and let $\tau_{2}$ be the infimum of times $t>\tau_{1}$ when $x(t)$ or $x(t-)$ is in $L-A$. In each case the stopping time is defined as $\infty$ if there is no time satisfying the stated conditions. Almost every sample function of this positive martingale either vanishes or has left limit 0 at time $\tau_{1}$ when $\tau_{1}<a$. But then it is well known (see for example [2]) that the martingale vanishes for $t>\tau_{1}$ and therefore $\tau_{2} \geqq a$ almost surely. Since $a$ is an arbitrary strictly positive number and $A_{1}$ is an arbitrary compact subset of $A$ the proof is complete under the stated hypotheses. In the unbounded case replace $g$ in the preceding discussion by $(g \wedge 1)_{0}$. The martingale then becomes a supermartingale. If $g$ is continuous this positive supermartingale vanishes or has left limit 0 at time $\tau_{1}$ when $\tau_{1}<a$, because $(g \wedge 1)_{0} \leqq g$, and this supermartingale becomes strictly positive near time $\tau_{2}$ when $\tau_{2}<a$ because $g$ is continuous, and in fact lower semicontinuity would be sufficient. Thus the theorem is valid for $g$ continuous. If $g$ is not continuous, the reasoning just used remains applicable if $A_{1}$ contains no branch point because then $x\left(\tau_{1}\right)$ is in $A_{1}$ almost surely whenever $x\left(\tau_{1}-\right)$ is in $A_{1}$, according to (2.2).

(7.7) Special exit laws. We have seen that a smooth $(f, 1)$ process with entrance state space $L$ has an almost sure limit at 0 if and only if $f$ has the form (3.3.1). The corresponding set of absolute probability functions for the dual transition function $p^{*}$ suggests that the class of exit laws for $p$ of the form

$$
g(t, i)=\int_{L^{*}} p_{h}\left(t, i, \xi^{*}\right) p_{0}\left(d \xi^{*}\right)
$$


where $L^{*}$ is an exit space and $p_{0}$ is a measure, which can be chosen if desired supported by the set of nonbranch points for $p^{*}$, will somehow play a dual role. This role will be exhibited in $\S 11$.

8. Space time excessive measures. In this section $L$ is always an exit space.

(8.1) Definition. For each $t>0$ let $f(t, \cdot)$ be a positive measure on $I$, and write $f(t, j)$ for $f(t,\{j\})(\leqq \infty)$. If $\alpha \geqq 0, f$ will be called a space time $\alpha$ supermedian measure if

$$
e^{-\alpha s} \sum_{i} f(t-s, i) p(s, i, j) \leqq f(t, j) \quad(0<s<t) .
$$

The left side increases as $s$ decreases and we denote the limit at 0 by $f_{0}$. If $f_{h}$ is defined by $f_{h}(t, i)=f(t, i) / h(i)$ (the Radon-Nikodym derivative of the measure $f(t, \cdot)$ with respect to the measure $h$ defined in (4.2.1)), and if we also set $f_{h}(t, \Delta)=0$, the function $(t, i) \leadsto e^{-t} f_{h}(t, i)$ is a space time $\alpha$ supermedian function for $I^{*}$ relative to the dual transition function $p^{*}$. If $f=f_{0}, f$ is called a space time $\alpha$ excessive measure, and is so if and only if $(t, i) \leadsto e^{-t} f_{h}(t, i)$ is a space time $\alpha$ excessive function for $p^{*}$. We can thus read off for example the following properties of $f_{h}$ (in the excessive case) from the corresponding properties of $\alpha$ excessive functions: $f(\cdot, i)$ is left continuous, $(t, i) \leadsto e^{-t} f_{h}(t, i)$ has a unique extension to a space time $\alpha$ excessive function for $p^{*}$ on $(0, \infty) \times L$. Since $L$ must be adapted to $p$ as well as to its dual, to every absolute probability function $f$ for $p$ corresponds a smooth $(f, 1)$ process $\{x(t), t>0\}$ (transition function $p$ ) with state space $L$. Now define $x_{0}^{*}(t)$ as in (4.3), so that $\left\{x_{0}^{*}(t+), t>0\right\}$ is smooth (transition function $p^{*}$ ). Then according to Theorem 6.8 (which is applicable to adapted state spaces if only strictly positive parameter values are considered) applied to the dual transition function, if $a>0$ the

$$
\left\{f_{h}\left[a-t, x^{*}(t+)\right], 0<t<a\right\}
$$

process is a right continuous process with left limits.

\section{Entrance laws.}

(9.1) Definition. For each $t>0$ let $f(t, \cdot)$ be a positive measure on $I$, and write $f(t, j)$ for $f(t,\{j\})(\leqq \infty)$. If

$$
\sum_{i} f(t, i) p(s, i, j)=f(s+t, j) \quad(s, t>0)
$$

$f$ will be called an entrance law. (Note that this definition omits Neveu's [4] boundedness condition.) An entrance law is a space time $\alpha$ excessive measure for every $\alpha \geqq 0$. If $\sum_{i} f(t, i)=1$, the entrance law $f$ is called an absolute probability function. If $f$ is an entrance law and if $f_{h}(t, i)=f(t, i) / h(i), f_{h}(t, \Delta)=0$, then, following (8.1), the function $(t, i) \leadsto e^{-t} f_{h}(t, i)$ is an exit law for the dual transition function $p^{*}$. Conversely if $g^{*}$ is an exit function for the dual transition function, with $g^{*}(\cdot, \Delta)=0,(t, i) \leadsto e^{t} g^{*}(t, i) h(i)$ is an entrance law for $p$. If $f$ is an entrance law and if $u$ is defined by

$$
u(i)=\int_{0}^{\infty} e^{-\alpha s} f(s, i) d s
$$


an argument exactly like that in $\S 7$ shows that $u$ is an $\alpha$ excessive measure, and

$$
\lim _{t \rightarrow \infty} e^{-\alpha t} \sum_{i} f(t, i) p(t, i, j)=0
$$

whenever $u(i)$ is finite. Conversely if $u$ is an $\alpha$ excessive measure, finite at each integer, and satisfies (9.1.2), there is a corresponding entrance law satisfying (9.1.1).

(9.2) Extension of an entrance law. If $f$ is an entrance law,

$$
\sum_{i} f_{h}(s, i) p_{h}(t-s, i, j) h(i)=f_{h}(t, j) \text {. }
$$

Let $L^{*}$ be an exit space and define $f_{h}(t, \cdot)$ on $L^{*}$ by the left side of $(9.2 .1)$, using the continuous extension of $p_{h}(\cdot, i, \cdot)$ to $(0, \infty) \times L^{*}$. This extension is a special case of the extension of a space time $\alpha$ excessive measure described in (8.1).

(9.3) THEOREM. If $L^{*}$ is an exit space and if $f$ is an entrance law, then for each $\xi^{*}$ in $L^{*}$ the function $f_{h}\left(\cdot, \xi^{*}\right)$ vanishes identically if at all. If $A$ is the set of zeros of $f_{h}(t, \cdot)$ almost no path of a smooth process with state space $L^{*}$ (transition function $p$ ) hits $A$ in a nonbranch point for $p^{*}$ or has a left limit in $A$ after hitting $L^{*}-A$ or having a left limit in $L^{*}-A$. If $f_{h}$ is continuous, in particular if $\sup _{t, i} e^{-t} f_{h}(t, i)<\infty$, the words 'nonbranch point for $p^{*}$ ' can be deleted.

This theorem is the dual of Theorem 7.6, and is proved by applying that theorem to the dual situation. The most interesting special case is $f=p(\cdot, \xi, \cdot)$ for $\xi$ a point of an entrance space.

(9.4) Extension of $p_{h}$. Let $L$ and $L^{*}$ be respectively entrance and exit adapted spaces. Then for each point $\xi$ of $L, p(\cdot, \xi, \cdot)$ is an entrance law. Denote by $p_{h}^{\prime}(\cdot, \xi, \cdot)$ the extension to $(0, \infty) \times L^{*}$ of the function $(t, i) \leadsto p_{h}(t, \xi, i)$ obtained in (9.2). Then

$$
\sum_{i} p_{h}^{\prime}(t, \xi, i) p_{h}\left(s, i, \xi^{*}\right) h(i)=p_{h}^{\prime}\left(s+t, \xi, \xi^{*}\right) \quad\left(\xi \in L, \xi^{*} \in L^{*}\right) .
$$

The dual of this extension is the following. For each point $\xi^{*}$ of $L^{*}, p_{h}\left(\cdot, \cdot, \xi^{*}\right)$ is an exit law. Denote by $p_{h}^{\prime \prime}\left(\cdot, \cdot, \xi^{*}\right)$ the extension (see $\left.(7.1)\right)$ to $(0, \infty) \times L$ of this law. Then

$$
\sum_{i} p(t, \xi, i) p_{h}^{\prime \prime}\left(s, i, \xi^{*}\right)=p_{h}^{\prime \prime}\left(s+t, \xi, \xi^{*}\right) .
$$

Our definitions imply that

$$
p_{h}^{\prime}(t, \xi, i) h(i)=p(t, \xi, i), \quad p_{h}^{\prime \prime}\left(s, i, \xi^{*}\right)=p_{h}\left(s, i, \xi^{*}\right) .
$$

Hence the left sides of (9.4.1) and (9.4.2) are equal and we have proved that $p_{h}^{\prime}=p_{h}^{\prime \prime}$. From now on this function will be denoted by $p_{h}$. It is lower semicontinuous on $(0, \infty) \times L \times L^{*}$ and satisfies

$$
\sum_{i} p(s, \xi, i) p_{h}\left(t, i, \xi^{*}\right)=p_{h}\left(s+t, \xi, \xi^{*}\right)
$$


This function $p_{h}$ is the version in the present context of Lumer-Naïms $\theta$ kernel in classical potential theory [3] in the context of the Martin boundary.

(9.5) Intrinsic pairs of entrance exit laws. Let $L$ be an entrance exit space, and let $L^{\prime}$ be a second entrance exit space, perhaps obtained using another choice $h^{\prime}$ of $h$, that is using another choice of the sequence $\left\{a_{n}, n \geqq 1\right\}$ in (4.2.1). We use our usual notation $p, p_{h}$ referring to $L$, and correspondingly $p^{\prime}, p_{h^{\prime}}^{\prime}$ for these functions based on $L^{\prime}$. Since $h^{\prime}$ and $h$ are both 1 excessive measures on $I, h^{\prime} / h$ (defined as 0 at $\Delta$ ) is a 0 excessive function on $I^{\Delta}$ relative to $p^{*}$. Then $h^{\prime} / h$ has a space time 0 excessive (relative to $p^{*}$ ) extension to $(0, \infty) \times L$, which reduces to a 0 excessive extension to $L$, and we keep the notation $h^{\prime} / h$ for this extension. Let $\xi$ be a regular point of $L$. Since $L^{\prime}$ is an entrance space there is (Theorem 3.9) a unique regular point $\xi^{\prime}$ of $L^{\prime}$ with $p(\cdot, \xi, \cdot)=p^{\prime}\left(\cdot, \xi^{\prime}, \cdot\right)$ on $(0, \infty) \times I$. Now let $\left\{x^{*}(t), t>0\right\}$ be a smooth process for the transition function $p^{*}$ with state space $L^{\Delta}$. Then the process $\left\{\left(h^{\prime} \mid h\right)\left[x^{*}(t)\right], t>0\right\}$ is a right continuous supermartingale if its expectations are finite, as they are if the $x^{*}$ process is chosen to have $x^{*}(0+)=i$ almost surely,

$$
E\left\{\left(h^{\prime} / h\right)\left[x^{*}(t)\right]\right\} \leqq h^{\prime}(i) / h(i) .
$$

Since $\xi$ is regular, $i$ can be chosen so that $\xi$ is hit with strictly positive probability by $x^{*}$ paths. Hence $\left(h^{\prime} / h\right)(\xi)<\infty$. If the $x^{*}$ process has absolute probability function $p^{*}(\cdot, \xi, \cdot)$, to make $x^{*}(0+)=\xi$ almost surely, (9.5.1) is true with $i$ replaced by $\xi$, so $h^{\prime} / h$ on the $x^{*}$ process is a supermartingale, in fact even for the parameter interval $[0, \infty)$ if we define $x^{*}(0)=\xi$. By $(7.5)$ applied to dual processes this supermartingale is right continuous at $t=0$. For $t$ restricted to be rational, the process $\left\{x^{*}(t), t>0\right\}$ can be considered a process with state space $I$, or $L^{\prime}$, and we have for $t$ rational, $s$ fixed, $i$ fixed,

$$
\frac{h^{\prime}\left[x^{*}(t)\right]}{h\left[x^{*}(t)\right]} p_{h^{\prime}}^{\prime}\left[s, i, x^{*}(t)\right]=p_{h}\left[s, i, x^{*}(t)\right] .
$$

When $t \rightarrow 0$ this yields, since $\xi^{\prime}$ is regular and therefore not a dual branch point,

$$
\left(h^{\prime} / h\right)(\xi) p_{h^{\prime}}^{\prime}\left(s, i, \xi^{\prime}\right)=p_{h}(s, i, \xi) .
$$

Thus the exit laws $p_{h}(\cdot, \cdot, \xi), p_{h^{\prime}}^{\prime}\left(\cdot, \cdot, \xi^{\prime}\right)$ are the same up to a constant factor.

We have now achieved the following results connecting the regular points of different state spaces. If $\xi$ is a point of an entrance [exit] space, $\xi$ generates an entrance law $p(\cdot, \xi, \cdot)$ [exit law $p_{h}(\cdot, \cdot, \xi)$ ], and if $\xi$ is a nonbranch point for $p\left[p^{*}\right]$ no other nonbranch point for $p\left[p^{*}\right]$ generates this law. In particular if $\xi$ is a regular point of an entrance exit space the point generates both an entrance and an exit law and some uniquely determined regular point of each entry exit space generates the same pair of laws, neglecting constant factors, and even allowing different choices of the normalizing measure $h$. Each member of the pair uniquely determines the other up to a constant factor. Moreover (Theorem 3.9) if $\xi$ is a regular point of any entrance [exit] space the entrance [exit] law $\xi$ generates is 
also generated, aside from a constant factor, by some regular point of each entrance [exit] space.

\section{Taboo probabilities.}

(10.1) Conventions. Let $L$ be an entrance exit space, let $\eta$ be a point of $L$ and let $\{x(t), t>0\}$ be a smooth $(p(\cdot, \eta, \cdot), 1)$ process with this state space. Let $\xi$ be a point of $L$, possibly the same as $\eta$, let $\tau(\leqq \infty)$ be the infimum of strictly positive times that an $x$ process path or path left limit hits $\xi$, and let $F_{\eta \xi}$ be the distribution function of $\tau$. If $\xi$ is not a branch point, $F_{n \xi}$ is unchanged if 'or path left limit' is omitted in the definition. If $\xi$ is a branch point almost no path hits $\xi$ and there is a monotone increasing sequence $\left\{\tau_{n}, n \geqq 1\right\}$ of stopping times, with limit $\tau$ such that $\tau_{n}<\tau$ for all $n$ whenever $\tau_{n}<\infty$. Denote by $p^{\xi}(t, \eta, j)$ the probability that a path with $x(t)=j$ has not hit $\xi$ or had left limit $\xi$ at any time $<t$. The notation $p^{* \xi}$ will denote the corresponding probability for the dual transition function.

In the following work the Laplace transform of a measure $d F$ will be denoted by $(d F)^{\sim}$ and the Laplace transform of a function $\phi$ will be denoted by $\phi$. Only strictly positive arguments of the transforms will be used. Note that $\tilde{p}_{h}(\cdot, i, j)$ is finite, in fact at most

$$
\left[1-p^{*}(t, j, \Delta)\right] e^{t} / h(i)=\int_{t}^{\infty} e^{t-s} f^{0}(s, j) d s /[h(i) h(j)] \leqq 1 /[h(i) h(j)],
$$

and $\tilde{p}_{h}(\lambda, i, \xi)$ is finite for $\lambda>1$ because $p_{h}(t, i, \xi) \leqq e^{t} / h(i)$.

(10.2) THEOREM. If $\xi$ and $\eta$ are points of the entrance exit space $L$,

$$
p(t, \eta, j)=\int_{0}^{t} p(t-s, \xi, j) d F_{n \xi}(s)+p^{\xi}(t, \eta, j) .
$$

The last term vanishes if $\xi=j$. For all integers $i, j, k$,

$$
\tilde{p}(\cdot, i, j)=\tilde{p}(\cdot, i, k) \tilde{p}(\cdot, k, j) / \tilde{p}(\cdot, k, k)+\tilde{p}^{k}(\cdot, i, j) .
$$

To prove the theorem consider the martingale $\{p[t-s, x(s), j], 0<s<t\}$, using the $x$ process defined in (10.1). Define the martingale at $s=0$ as $p(t, \eta, j)$. Since $x(s)=k$ almost surely for values of $s$ arbitrarily close to $t$, when $x(t)=k$, this martingale has as limit at $t$ the indicator function of the set where $x(t)=j$. Adjoin this limit to the martingale to obtain a martingale with parameter interval $[0, t]$. If $\xi$ is not a branch point and if $\tau$ is defined as in (10.1), apply the martingale equality for expectations at times 0 and $t \wedge \tau$ to get (10.2.1). If $\xi$ is a branch point define $\tau_{n}$ as in (10.1), apply the martingale equality at times 0 and $t \wedge \tau_{n}$ and then when $n \rightarrow \infty$ get (10.2.1). It is trivial that the last term of (10.2.1) vanishes when $\xi=j$. To prove (10.2.2) write (10.2.1) with $\eta, \xi, j$ successively replaced by $i, k, j$ and $i, k, k$.

(10.3) THEOREM. If $\xi$ is a nonbranch point of the entrance exit space $L$,

$$
p_{h}(t, i, \xi)=\int_{0}^{t} p_{h}(t-s, \xi, \xi) d F_{i \xi}(s)+e^{t} p^{* \xi}(t, \xi, i) / h(i)
$$


If $\xi$ is regular, $\tilde{p}_{n}(\cdot, \eta, \xi) \leqq \tilde{p}_{h}(\cdot, \xi, \xi)<\infty$ for all $\eta$ in $L$, the last term of $(10.3 .1)$ vanishes, and

$$
\tilde{p}(\cdot, i, j)=\tilde{p}_{h}(\cdot, i, \xi) \tilde{p}(\cdot, \xi, j) / \tilde{p}_{h}(\cdot, \xi, \xi)+\tilde{p}^{\xi}(\cdot, i, j) .
$$

If $\xi=k$, (10.3.2) reduces to (10.2.2). Take $\eta=i$ and apply the same $x$ process and stopping time $\tau$ as in the proof of Theorem 10.2, setting $x(0)=i$. Since $p_{h}(\cdot, \cdot, \xi)$ is an exit law, the process $\left\{p_{h}[t-s, x(s), \xi], 0 \leqq s<t\right\}$ is a right continuous martingale. If $0<\delta<t$ the martingale equality for times 0 and $\tau \wedge(t-\delta)$ is

$$
\begin{aligned}
p_{h}(t, i, \xi) & =E\left\{p_{h}(t-\tau, \xi, \xi) 1_{\{\tau<t-\delta\}}\right\}+E\left\{p_{h}[\delta, x(t-\delta), \xi] 1_{\{\tau>t-\delta\}}\right\} \\
& =\int_{0}^{t-\delta} p_{h}(t-s, \xi, \xi) d F_{i \xi}(s)+\sum_{k} p^{\xi}(t-\delta, i, k) p_{h}(\delta, k, \xi) .
\end{aligned}
$$

The sum becomes, when expressed in terms of the dual transition function,

$$
\frac{e^{t}}{h(i)} \sum_{k} p^{*}(\delta, \xi, k) p^{* \xi}(t-\delta, k, i)
$$

If $\left\{x^{*}(t), t>0\right\}$ is a smooth $\left(p^{*}(\cdot, \xi, \cdot), 1\right)$ process for the transition function $p^{*}$, with state space $L$, the sum in (10.3.4) is the probability that $x^{*}(t)=i$ and that an $x^{*}$ process path never hits $\xi$ between times $\delta$ and $t-\delta$. When $\delta \rightarrow 0$ this probability becomes $p^{* \xi}(t, \xi, i)$. Therefore (10.3.1) is true. Suppose now that $\xi$ is regular. Then the last term in (10.3.1) vanishes because (Proposition 5.5) $\xi$ is also regular for $p^{*}$, and (10.3.1) becomes, in terms of Laplace transforms,

$$
\tilde{p}_{h}(\lambda, i, \xi)=\tilde{p}_{h}(\lambda, \xi, \xi)\left(d F_{i \xi}\right)^{\sim} .
$$

Since the left side is finite for $\lambda>1$ (see $(10.1)) \tilde{p}_{h}(\lambda, \xi, \xi)$ is also because (regularity of $\xi) i$ can be chosen to make $\left(d F_{i \xi}\right)^{\sim}$ strictly positive. Moreover according to (10.3.5),

$$
\tilde{p}_{h}(\lambda, i, \xi) \leqq \tilde{p}_{h}(\lambda, \xi, \xi)
$$

for $\lambda>1$ and all $i$. Then

$$
\int_{0}^{t} p_{h}(s, i, \xi) d s \leqq e^{2 t} \tilde{p}_{h}(2, \xi, \xi)<\infty
$$

and therefore

(10.3.8) $\sum_{j} p(n, i, j) \int_{0}^{1} p_{h}(s, j, \xi) d s=\int_{n}^{n+1} p_{h}(s, i, \xi) d s \leqq e^{2} \tilde{p}_{h}(2, \xi, \xi)$

from which we conclude that $\tilde{p}_{h}(\lambda, i, \xi)$ is finite for all $\lambda>0$. We can now conclude that (10.3.5) implies the finiteness of $\tilde{p}_{h}(\lambda, \xi, \xi)$ for all $\lambda>0$ and that $\tilde{p}_{h}(\cdot, i, \xi)$ $\leqq \tilde{p}_{h}(\cdot, \xi, \xi)$. Hence the same inequality is true if $i$ is replaced by an arbitrary point of $L$. Finally if $(10.3 .5)$ is used to eliminate $\left(d F_{i \xi}\right) \sim$ in the Laplace transform version of (10.2.1) we obtain (10.3.2). 
(10.4) Neveu coupled laws. Let $L$ be an entrance exit space. If $\xi$ is a regular point of $L,(10.3 .2)$ implies that

$$
\tilde{p}(\cdot, i, j) \geqq \tilde{p}_{h}(\cdot, i, \xi) \tilde{p}(\cdot, \xi, j) / \tilde{p}_{h}(\cdot, \xi, \xi) .
$$

If $f$ and $g$ are respectively entrance and exit laws and if $0<s<t$ define

$$
[f, g](t)=\sum_{k} f(s, k) g(t-s, k) .
$$

The sum does not depend on $s$. Let $[f, g]^{\sim}$ be the Laplace transform of $[f, g]$. Neveu [4] studied pairs $f, g$ of entrance and exit laws satisfying

$$
\sum_{i} f(t, i)<\infty, \quad \sup _{i} \int_{0}^{t} g(s, i) d s<\infty
$$

for all (equivalently some) $t>0$ and

$$
\tilde{p}(\cdot, i, j) \geqq \tilde{g}(\cdot, i) \tilde{f}(\cdot, j) /[f, g]^{\sim} .
$$

It is clear, using (10.3.7), that $p(\cdot, \xi, \cdot), p_{h}(\cdot, \cdot, \xi)$ is such a pair when $\xi$ is regular. Conversely if $f, g$ is any such pair, Williams [5] showed that (aside from an irrelevant constant of proportionality) $f=p(\cdot, \xi, \cdot)$, for $\xi$ some regular point of a canonical entrance space, equivalently for $\xi$ some regular point of any entrance space according to Theorem 3.9. Neveu showed that $g$ is uniquely determined by $f$ (aside from a constant of proportionality) and called each such pair with $\xi$ not an integer a 'fictitious event'. (Two pairs are considered the same if corresponding elements differ only by factors of proportionality.) Williams obtained the second member of a pair corresponding to the regular point $\xi$ in terms of the local time at $\xi$. The Neveu pairs are evidently the pairs of entrance and exit laws generated by the regular points of any entrance exit space, as discussed in (9.5).

11. $(f, g)$ process.

(11.1) Distribution of an $(f, g)$ process. Let $f$ and $g$ be respectively entrance and exit laws satisfying

$$
\sum_{i} f(t, i)=1, \quad 0<[f, g](t)<\infty .
$$

Choose $b>0$ and define probabilities for a process $\{x(t), 0<t<b\}$ with state space $I$ by

$$
\begin{aligned}
P\left\{x\left(t_{1}\right)\right. & \left.=i_{1}, \ldots, x\left(t_{n}\right)=i_{n}\right\} \\
& =f\left(t_{1}, i_{1}\right) p\left(t_{2}-t_{1}, i_{1}, i_{2}\right) \cdots p\left(t_{n}-t_{n-1}, i_{n-1}, i_{n}\right) g\left(b-t_{n}, i_{n}\right) /[f, g](b)
\end{aligned}
$$

for $0<t_{1}<\cdots<t_{n}<b$. This distribution is Markovian with absolute probability function given by

$$
P\{x(t)=i\}=f(t, i) g(b-t, i) /[f, g](b)
$$


and transition function (from $i$ at time $s$ to $j$ at time $t>s$ ) given by

$$
p(t-s, i, j) g(b-t, j) / g(b-s, i) .
$$

A zero of the denominator in (11.1.4) causes no trouble because $P\{x(s)=i\}=0$ when $g(b-s, i)=0$. A process with parameter interval $(0, b)$ and the finite dimensional distribution (11.1.2) will be called an $(f, g)$ process. If $L$ is a topological space containing $I$, an $(f, g)$ process with state space $L$, right continuous with left limits, will be called a smooth $(f, g)$ process. If $j$ is an integer and $0<b_{1}<b,(f, g)$ process probabilities involving parameter values $\leqq b_{1}$, with $x\left(b_{1}\right)=j$, are the same as those for $g=1$ except for the factor $g\left(b-b_{1}, j\right) /[f, g](b)$. Thus if $L$ is a topological space adapted to $p$ there is a smooth $(f, g)$ process with state space $L$.

If $g=1$ the value of $b$ is irrelevant and can be taken as $\infty$, and an $(f, g)$ process then becomes an $(f, 1)$ process, as defined earlier, if, as we can suppose, $\sum_{i} f(t, i)=1$.

(11.2) Extension of theorems on $(f, 1)$ processes to $(f, g)$ processes. If $L$ is an entrance space, if $\{x(t), t>0\}$ is a smooth $(f, g)$ process with state space $L$, if $g^{\prime}$ is a space time $\alpha$ excessive function and if $a>0$ it was seen in $\S 6$ that when $g=1$ the process $\left\{g^{\prime}[a-t, x(t)], 0<t<a\right\}$ is right continuous with left limits. The argument used to derive the continuity properties of $(f, g)$ process sample functions shows that the earlier result is true for general $g$, with of course the restriction that $0<t<a \wedge b$. In particular we can take $g^{\prime}=g$. We shall use the fact that almost every sample function of the process $\{g[a-t, x(t)], 0<t<a \wedge b\}$ is bounded away from 0 on each compact subinterval of the parameter interval. To see this note first that the process

$$
\left\{e^{-\alpha t}\left(g^{\prime} / g\right)[b-t, x(t)], 0<t<b\right\}
$$

is a supermartingale if the random variables have finite expectations, as they do if $g^{\prime}=1$. In that case the process is a right continuous positive supermartingale and as such has sample functions which are almost all bounded on compact parameter intervals, as was to be proved. Note that this means that the process (11.2.1) is right continuous with left limits.

The theorems in previous sections for $(f, 1)$ processes go over into theorems for $(f, g)$ processes, just as sample function smoothness did; alternatively proofs can be given valid for all $g$. Thus (Theorem 7.6) if $L$ is an entrance space, if $g^{\prime}$ is an exit law, and if $A$ is the set of zeros of $g^{\prime}$, almost no path of any smooth $(f, g)$ process with state space $L$ which ever hits $A$ or has left limit in a nonbranch point of $A$ or has limit in $A$ at $t=0$ ever hits $L-A$ or has left limit in $L-A$ thereafter. To give a proof valid for all $g$ replace the process $\left\{g^{\prime}[a-t, x(t)], 0<t<b\right\}$ which would be used in the proof when $g=1$ by the (right continuous) process

$$
\left\{g^{\prime}[a-t, x(t)] / g[b-t, x(t)], 0<t<a \wedge b\right\},
$$

or by the minimum with $c$ if the expectations of these random variables are not finite. If the expectations are finite the process is a martingale. 
(11.3) $(f, g)$ processes at 0 . Let $L$ be an entrance space, let $f$ be an absolute probability function of the form (3.3.1) and let $\{x(t), t>0\}$ be a smooth $(f, g)$ process with state space $L$. Then according to the principle of converting $(f, 1)$ into $(f, g)$ probabilities stated in $(11.1), x(0+)$ exists almost surely and has distribution $g(b, \eta) f(0+, d \eta) /[f, g](b)$. In more detail on this last point, the probability that $x(0+)$ lies in $A$ is the probability that $x(0+)$ lies in $A$ and that $x(b / 2)=j$, summed over $j$ :

$$
\sum_{j} \int_{A} p(b / 2, \eta, j) f(0+, d \eta) g(b / 2, j) /[f, g](b)=\int_{A} g(b, \eta) f(0+, d \eta) /[f, g](b) .
$$

In particular if $\xi$ is not a branch point and if $f=p(\cdot, \xi, \cdot)$, the limit $x(0+)$ is almost surely $\xi$, just as in the case $g=1$.

(11.4) $(f, g)$ processes at $b$. The dual of the results in (11.3) is the following. Let $L^{*}$ be an exit space, let $g$ be an exit law of the form (7.7.1). Then if $\{x(t), t>0\}$ is a smooth $(f, g)$ process with state space $L^{*}$ (and we know there is one because $L^{*}$ is adapted to $\left.p\right), x(b-)$ exists almost surely. To see this we need only note that the formula (11.1.2) is completely symmetric in the following sense. If it is written in terms of $p^{*}$ and if one uses the fact that the function $(t, i) \leadsto \rightarrow e^{-t} f_{h}(t, i)$ is an exit law for $p^{*}$ and that $(t, i) \leadsto e^{-t} g(t, i) h(i)$ is an entrance law for $p^{*}$, then the formula (11.1.2) goes over into the corresponding formula with time reversed. That is, an $(f, g)$ process relative to $p$ under time reversal becomes an $\left(f^{*}, g^{*}\right)$ process for $p^{*}$, where $f^{*}(t, i)=e^{-t} g(t, i) h(i), g^{*}(t, i)=e^{-t} f_{h}(t, i)$. The distribution of $x(b-)$ is the limiting distribution $(t \rightarrow 0)$ of the distribution assigning measure

$$
e^{-t} \int_{L^{*}} p_{h}\left(t, i, \xi^{*}\right) h(i) p_{0}\left(d \xi^{*}\right)
$$

to the singleton $\{i\}$. In particular if $\xi^{*}$ is not a branch point for $p^{*}$ and if $g=p\left(\cdot, \cdot, \xi^{*}\right)$, the value of $x(b-)$ is almost surely $\xi^{*}$.

(11.5) Processes from point to point. Let $L$ be an entrance exit space, and let $\xi$ be a regular point of $L$. Then $\xi$ is not a branch point for $p^{*}$. Let $\eta$ be a nonbranch point for $p$, chosen so that with strictly positive probability a smooth $(p(\cdot, \eta, \cdot), 1)$ path hits $\xi$. (For example choose $\eta=\xi$.) Applying Theorem 7.6 it follows readily that $p_{h}(\cdot, \eta, \xi)>0$. Moreover $p_{h}(t, \eta, \xi)<\infty$ for (Lebesgue) almost every $t$ because (Theorem 10.3) $\tilde{p}_{h}(\cdot, \eta, \xi)<\infty$, and we choose $b$ with $p_{h}(b, \eta, \xi)<\infty$. If now $f=p(\cdot, \eta, \cdot)$ and $g=p_{h}(\cdot, \cdot, \xi)$ then $0<[f, g](b)=p_{h}(b, \eta, \xi)<\infty$ and a smooth $(f, g)$ process with state space $L$ has almost surely limit $\eta$ at $t=0$, limit $\xi$ at $t=b$. Such a process, with $\xi=\eta$, has been used in a different context by Neveu [4].

\section{BIBLIOGRAPHY}

1. K. L. Chung, Markov chains with stationary transition probabilities, 2nd ed., Die Grundlehren der math. Wissenschaften, Band 104, Springer-Verlag, Berlin and New York, 1967. MR 36 \#961. 
2. J. L. Doob, Compactification of the discrete state space of a Markov process, Z. Wahrscheinlichkeitstheorie und Verw. Gebiete 10 (1968), 236-251. MR 38 \#2842.

3. L. Lumer-Naïm, Sur le rôle de la frontière de R. S. Martin dans la théorie du potentiel, Ann. Inst. Fourier Grenoble 7 (1957), 183-285.

4. J. Neveu, Sur les états d'entrée et les états fictifs d'un processus de Markov, Ann. Inst. H. Poincaré 17 (1962), 323-337. MR 33 \#784.

5. David Williams, Fictitious states, coupled laws and local time, Z. Wahrscheinlichkeitstheorie und Verw. Gebiete 11 (1969), 288-310.

UNIVERSITY OF ILLINOIS,

URBANA, ILLINOIS 61801 